\title{
Auf dem Weg zu einer Gesundheitsförderung mit Kopf, Herz und Hand
}

\section{Mathis Brauchbar}

advocacy ag

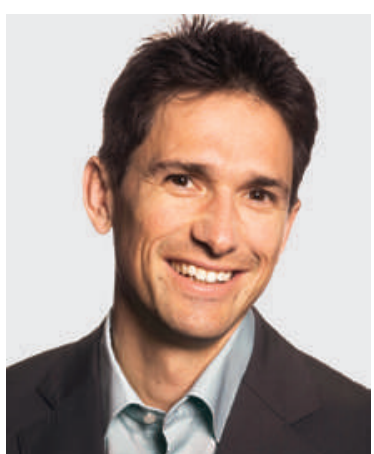

Thomas Mattig, Direktor Gesundheitsförderung Schweiz: «In einer gerechten Gesellschaft ist Gesundheitsförderung darauf ausgerichtet, die Autonomie der Individuen und die Gleichheit in der Gesellschaft in eine ausgewogene Beziehung zu bringen.»
Korrespondenz:

Dr. Thomas Mattig

Gesundheitsförderung Schweiz Dufourstrasse 30

CH-3000 Bern 6

thomas.mattig[at]

promotionsante.ch
Die 15. Nationale Gesundheitsförderungs-Konferenz steht unter dem Titel «Autonomie als Herausforderung für $\mathrm{Ge}$ sundheitsförderung und Prävention». Weshalb thematisiert die Konferenz einen eher philosophischen Begriff wie die Autonomie?

Thomas Mattig: Der Gesundheitsförderung wie auch der Prävention werden in der politischen Debatte immer wieder vorgeworfen, die persönlichen Freiheiten und die Selbstbestimmtheit der Menschen einzuschränken. Tatsächlich befindet sich aktive Gesundheitsförderung hier in einem Spannungsfeld: Einerseits möchte sie gesunde Lebensstile ermöglichen, andererseits greift sie dafür auch lenkend in die Gesellschaft ein. Dieses Spannungsfeld möchten wir an unserer Konferenz ausleuchten und diskutieren.

\section{Können Sie ein konkretes Beispiel geben?}

Eines der Ziele der Gesundheitsförderung ist die Reduktion der Fettleibigkeit in der Bevölkerung. Um dies zu erreichen, genügt es nicht, einfach an gesunde Ernährung und viel Bewegung zu appellieren. Wir müssen diesen Appell auch unterstützen, etwa durch Vermittlung von Wissen in den Schulen oder durch die Förderung von Verhältnissen, die einen gesunden Lebensstil ermöglichen, beispielsweise durch Massnahmen im Verkehr, damit Kinder zu Fuss zur Schule gehen können. Dadurch bewegen wir uns in einem politischen Spannungsfeld.

\section{Ist Gesundheitsförderung Politik?}

Teilweise. Indem Gesundheitsförderung auf Lebensstile und -bedingungen abzielt, kann sie als politisches Programm betrachtet werden. Der Ursprung der Gesundheitsförderung, wie wir sie heute kennen, ist denn auch nicht in der Praxis von Public Health oder in der Präventionsmedizin zu finden. Gesundheitsförderung ist vielmehr «top down» ent-

\footnotetext{
Autonomie als Herausforderung für die Gesundheitsförderung und Prävention

15. Nationale Gesundheitsförderungs-Konferenz 2014, am 30. Januar 2014 im Kongresszentrum Beaulieu Lausanne. Programm und Anmeldung unter: www.gesundheitsfoerderung.ch/konferenz
}

standen. Sie geht zurück auf Prozesse innerhalb der Weltgesundheitsorganisation WHO: Mit der Gründung der WHO wurde Gesundheit zu einem völkerrechtlich verbrieften Recht und ihre Förderung zur staatlichen Pflicht.

Mit der Ottawa-Charta von 1986 wurde die Gesundheitsförderung zu einem politischen Programm?

Die Ottawa-Charta setzt einige Normen, welche die Tätigkeit der Gesundheitsförderung entscheidend prägen, die aber kaum diskutiert und noch weniger legitimiert werden. Im Zentrum stehen die Normen Chancengleichheit und Autonomie. Gleichheit und Autonomie können jedoch zu Widersprüchen führen, weil sie unterschiedliche Ziele verfolgen. In einer gerechten Gesellschaft ist daher Gesundheitsförderung immer darauf ausgerichtet, die Autonomie der Individuen und die Gleichheit in der Gesellschaft in eine ausgewogene Beziehung zu bringen.

\section{Welche Folgen hat dies?}

Wir verfügen heute über kein umfassendes Modell, auf das sich die Gesundheitsförderung stützen könnte. Gesundheitsförderung ist vielmehr eine Strategie, die darauf zielt, die Gesundheit und das Wohlbefinden in der Gesellschaft zu verbessern. Das Fehlen einer theoretischen Grundlage hat seine Vorteile: Gesundheitsförderer und -förderinnen sind Praktiker und Pragmatiker. Sie ergehen sich nicht in langen Theorien, sondern packen dort an, wo es nötig ist, Gleichzeitig bewirkt das Fehlen eines Modells im Alltag Probleme: Weder das Konzept der Salutogenese noch jenes der Gesundheitsdeterminanten werden konsequent umgesetzt.

\section{Und was wäre aus Ihrer Sicht zu tun?}

Es ist an der Zeit, das theoretische Fundament der Gesundheitsförderung zu diskutieren und aufzubauen. Das ist gegenwärtig eine der wichtigsten Aufgaben von Gesundheitsförderung, denn sie legt die Basis für künftiges Arbeiten und politisches Durchsetzungsvermögen. Gleichzeitig darf die Gesundheitsförderung ihre pragmatische und praktische Herkunft nicht vergessen. Damit Gesundheitsförderung noch besser wird, mit Theorie und Praxis, mit Kopf, Herz und Hand. 